\title{
Attenuation of N2 amplitude of laser-evoked potentials by theta burst stimulation of primary somatosensory cortex
}

\author{
Csaba Poreisz $\cdot$ Andrea Antal · Klára Boros • \\ Nadine Brepohl · Gábor Csifcsák · Walter Paulus
}

Received: 16 August 2007 / Accepted: 17 October 2007 / Published online: 28 November 2007

(C) The Author(s) 2007

\begin{abstract}
Theta burst stimulation (TBS) is a special repetitive transcranial magnetic stimulation (rTMS) paradigm, where bursts of low-intensity stimuli are applied in the theta frequency. The aim of this study was to investigate the effect of neuronavigated TBS over primary somatosensory cortex (SI) on laser-evoked potentials (LEPs) and acute pain perception induced with Tm : YAG laser stimulation. The amplitude changes of the N1, N2, and P2 components of LEPs and related subjective pain rating scores of 12 healthy subjects were analyzed prior to and following continuous TBS (cTBS), intermittent TBS (iTBS), intermediate TBS (imTBS), and sham stimulation. Our results demonstrate that all active TBS paradigms significantly diminished the amplitude of the N2 component, when the hand contralateral to the site of TBS was laser-stimulated. Sham stimulation condition had no significant effect. The subjective pain perception also decreased during the experimental sessions, but did not differ significantly from the sham stimulation condition. The main finding of our study is that TBS over SI diminished the amplitude of the N2 component evoked from the contralateral side without any significant analgesic effects. Furthermore, imTBS produced responses similar to those observed by other forms of TBS induced excitability changes in the SI.
\end{abstract}

C. Poreisz $(\bowtie) \cdot$ A. Antal · K. Boros · N. Brepohl ·

G. Csifcsák · W. Paulus

Department of Clinical Neurophysiology,

Georg-August University of Göttingen,

Robert Koch Strasse 40, 37075 Göttingen, Germany

e-mail: csaba.poreisz@med.uni-goettingen.de

G. Csifcsák

Department of Psychiatry, University of Szeged,

Semmelweis u. 6, 6725 Szeged, Hungary
Keywords Theta burst transcranial magnetic stimulation . Primary somatosensory cortex $\cdot$ Laser-evoked potentials . Pain $\cdot$ Neuronavigation

\section{Introduction}

Functional neuroimaging studies have provided unequivocal evidence of the participation of the primary somatosensory cortex (SI), secondary somatosensory cortex (SII), and insula in pain processing (Talbot et al. 1991; Casey et al. 1994; Apkarian et al. 1999; Gelnar et al. 1999). Evidence suggests that the nociceptive input into these regions at least partially underlies the perception of sensory features of pain (Bushnell et al. 1999; Coghill et al. 1999; Peyron et al. 1999; Chen et al. 2002; for a reviews see: Peyron et al. 2000; Apkarian et al. 2005).

Electrophysiological studies have also confirmed the participation of the SI in pain processing, contralateral to the stimulated side. Tarkka and Treede (1993) first reported pain induced-activity in the SI using electroencephalogram (EEG) and applying brain electric source analysis (BESA). In their model, the peak latency measured at the SI was very similar to that of SII. In a combined magnetoencephalogramm (MEG) and laser-evoked potential (LEP) study Ploner et al. (1999) reported SI activity also contralateral to the side of stimulation and further to this proposed a parallel pain processing in SI and SII. This parallel activation of SI and SII was confirmed by other studies (Ploner et al. 2000, 2002) and other groups (Timmermann et al. 2001; Kanda et al. 2000; Inui et al. 2003; Nakata et al. 2004). However, some previous LEP dipole modelling studies showed that a dipole source in SI area was necessary to explain the scalp LEP topography, none of them reported a clear correspondence between the SI activity and a definite 
LEP component (Tarkka and Treede 1993; Ploner et al. 2002; Kanda et al. 2003). Kanda et al. (2000) detected SI activity following painful stimulation by recording intracranial EEG, but recently, intracerebral depth recordings in an epileptic patient have shown no reliable LEP response from the area $3 b$ of the SI after painful laser stimulation, although a reliable N2-P2 response could be recorded at $\mathrm{Cz}$ (Valeriani et al. 2004). Inui et al. (2003) reported the absence of activation from area $3 b$ of the SI after noxious electrical stimulation as well, however they found activity in the area 1 of SI. Other studies found no pain-related activation of the SI at all (for a review see: Garcia-Larrea et al. 2003; Kakigi et al. 2005).

Recent studies showed, that several kinds of external stimulation methods such as single-pulse transcranial magnetic stimulation (TMS) (Kujirai et al. 1993), $1 \mathrm{~Hz}$ repetitive TMS (rTMS) (Enomoto et al. 2001), paired associative stimulation (PAS) (Tsuji and Rothwell 2002; Wolters et al. 2005), transcranial direct current stimulation (tDCS) (Matsunaga et al. 2004; Dieckhofer et al. 2006) or theta burst stimulation (TBS) (Ishikawa et al. 2007) modulate the amplitude of cortical components of median nerve somatosensory evoked potentials (SEPs). It was recently reported that, cathodal tDCS over the SI (Dieckhofer et al. 2006) significantly reduced the N20 amplitude of median nerve SEPs. Furthermore, cathodal polarization over the SI induced a prolonged decrease of tactile discrimination (Rogalewski et al. 2004) and diminished acute pain perception and the amplitude of the N2 component of LEPs (Antal et al. 2007).

Recently Huang et al. (2005) developed a special "theta burst" paradigm to modulate human motor cortex (M1) excitability using low intensity, repetitive bursts of magnetic stimuli. The authors distinguished three stimulation patterns, which were proved to have different effects over M1 activity, when it was monitored by the amplitude of transcranial motor evoked potentials (MEPs). Continuous TBS (cTBS) caused a significant reduction in MEP amplitudes, which was probably due to the inhibition of specific excitatory circuits (I1-wave inputs to corticospinal neurons), as later confirmed by another study (Di Lazzaro et al. 2005). In contrast, intermittent TBS (iTBS) facilitated M1 activity and produced increase in MEP amplitudes. Interestingly, intermediate TBS (imTBS) had no effect at all. In addition, TBS has also been shown to have an effect on the human premotor (Mochizuki et al. 2005) and visual cortex (Franca et al. 2006).

Non-invasive cortical stimulation of M1 for the treatment of certain kinds of chronic and experimentally induced pain has recently attracted much interest. Both low and high frequency rTMS was reported to reduce subjective pain perception and has been used experimentally to reduce chronic pain (for reviews see: Leo and Latif 2007; Fregni et al. 2007). However, although the involvement of SI in pain perception and processing is reported by several imaging studies (for reviews see: Peyron et al. 2000; Apkarian et al. 2005), the magnetic stimulation of this cortical area in order to modify pain perception is neglected. Therefore, the aim of our study was to investigate the effects of cTBS, iTBS, and imTBS, on the early (N1) and late (N2, P2) components of LEPs and related subjective pain perception when applied over the left SI. We hypothesized that the three TBS types, which have short durations (maximum $190 \mathrm{~s}$ ), would affect LEP components in a specific, paradigm-dependent manner similarly to the effect over M1 as revealed by Huang and et al. (2005).

\section{Methods}

Subjects

Nineteen healthy volunteers between 18 and 35 years were informed about all aspects of the experiments and signed an informed consent. Three subjects chose not to continue the experiment after the first or second session and during offline EEG analysis four further subjects were dropped out because of their hardly detectable LEP components. Hence, twelve of the subjects (six male, six female; mean age $=26.33 \pm 3.17$ years) were included in the final analysis. We conformed with the Declaration of Helsinki and the experimental protocol was approved by the Ethics Committee of the University of Göttingen. None of the subjects suffered from chronic pain syndromes, nor took any medication regularly. None had a history of neurological or psychiatric illness. All of them participated in all four sessions, the three TBS and sham stimulations.

\section{TBS stimulation}

Theta burst stimulation was applied over the hand area of the left SI using a standard, figure-of-eight-coil (MCF-B65 Butterfly Coil) and MagPro stimulator (Medtronic, Denmark) with an outer half-coil radius of $75 \mathrm{~mm}$, with a posterior-anterior-posterior current flow in the coil. Stimulus intensity was $80 \%$ of active motor threshold (AMT) (Huang et al. 2005).

For AMT determination, the coil was placed tangentially to the scalp, with the handle pointing backwards and laterally $45^{\circ}$ from mid-line. MEPs of the right abductor digiti minimi muscle (ADM) were recorded by Ag-AgCl-electrodes in a belly tendon-montage before each stimulation. The signals were amplified and filtered $(1.59 \mathrm{~Hz}-1 \mathrm{kHz}$, sampling rate of $5 \mathrm{kHz}$ ), digitalized with a micro $1401 \mathrm{AD}$ converter (Cambridge Electronic Design, Cambridge, UK), recorded by a computer using SIGNAL software 
(Cambridge Electronic Design, Version 2.13). Complete muscle relaxation was controlled though auditory and visual feedback of EMG activity. AMT was defined as the minimum intensity eliciting a MEP of a superior size when compared to spontaneous moderate muscular activity in at least three of six pulses.

The pattern of TBS consisted of bursts containing three pulses at $50 \mathrm{~Hz}$ which were repeated at $200 \mathrm{~ms}$ intervals (i.e., $5 \mathrm{~Hz}$ ) for up to 600 pulses for $40 \mathrm{~s}$ continuously (cTBS), or triads repeated at $200 \mathrm{~ms}$ intervals for $2 \mathrm{~s}$ intermittently with $8 \mathrm{~s}$ breaks for up to 600 pulses (iTBS). In the case of imTBS the triads (three pulses at $50 \mathrm{~Hz}$ ) were repeated at $200 \mathrm{~ms}$ intervals for $5 \mathrm{~s}$, in every $15 \mathrm{~s}$ up to 600 pulses (Huang et al. 2005). In separate experimental sessions, sham stimulation was applied with the cTBS protocol using the same coil held over the same position but tilted to a $90^{\circ}$ angle (one-wing $90^{\circ}$ ) (Lisanby et al. 2001) with only the margin of the coil in contact with the scalp.

Determination of the primary somatosensory cortex (SI)

Anatomical magnetic resonance imaging (MRI) (Siemens 3 T, T1 weighted) dataset was used to determinate the coil localization for the stimulation of SI for all subjects using the Brainsight neuronavigation system (Rogue Research Inc., Montreal Quebec, Canada). The hand area was determined by previous fMRI studies (Bushnell et al. 1999; Blankenburg et al. 2003) and was located and marked in the MRI dataset as the target for TMS application (Fig. 1).

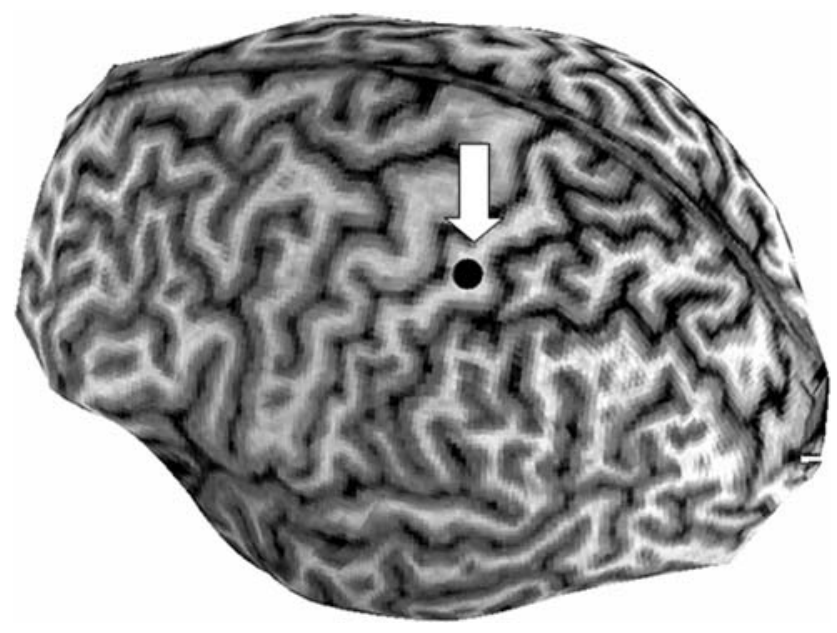

Fig. 1 Three dimensional anatomical MRI of a single subject. Anatomical magnetic resonance imaging $(M R I)$ (Siemens $3 \mathrm{~T}$, T1 weighted) dataset was used to determinate the coil localization for the stimulation of SI by all subjects using the Brainsight neuronavigation system. The hand area was located and marked in the MRI dataset as target for TMS application. The black point indicates the hot-spot of the stimulation coil
Laser stimulation

A Tm : YAG laser system (WaveLight Laser Technologie AG, Erlangen, Germany) was used to induce painful stimulation. The thulium laser emits near-infrared radiation (wavelength 2,000 $\mathrm{nm}$, pulse duration $1 \mathrm{~ms}$, laser beam diameter $7 \mathrm{~mm}$ ) with a penetration depth of $360 \mu \mathrm{m}$ into the human skin and allows a precise restriction of the emitted heat energy to the termination area of primary nociceptive afferents without affecting the subcutaneous tissue (Treede et al. 2003). The distal handpiece of the laser was positioned $30 \mathrm{~cm}$ from the radial part of the dorsal surface of the hand. The pain threshold was determined on both hands at the beginning each session before baseline EEG recording by applying laser stimuli from $200 \mathrm{~mJ}$ in $50 \mathrm{~mJ}$ steps. During EEG recordings, each laser stimulus was delivered with an intensity of 1.4-1.6 times the threshold intensity to a slightly different spot in a $5 \times 5 \mathrm{~cm}$ square on the dorsum of the hand in order to reduce receptor fatigue or sensitization by skin overheating (Treede et al. 2003). Skin temperature of the stimulated area was checked prior to every switch between hands, and corrected with a heating lamp if it fell below $35^{\circ} \mathrm{C}$.

Psychophysical evaluation

We used the verbal analogue score (VAS) to assess the subjective intensity of pain. The subjects were instructed to pay attention to the laser stimuli and to rate the perceived pain verbally (1-warm, 1.1 smallest pain, and 1.9 most intense pain) about 2-3 s after each laser-impulse. The values were individually averaged separately for both hands in each session and conditions. The ears of the subjects were plugged during the measurements to avoid auditory artifacts accompanying laser stimulation.

\section{Electrophysiological recordings}

The EEG was recorded using a 64-channel montage applying 64 ring electrodes (inner diameter: $6 \mathrm{~mm}$, outer diameter: $12 \mathrm{~mm}$ ) (EasyCap; Falk and Minow GmbH, Münich, Germany). The electrodes were placed in accordance with the extended international 10-20 system. The impedance was kept $<5 \mathrm{k} \Omega$. The Fz was used as reference, the ground was placed $2 \mathrm{~cm}$ anterior to the tragus of the right ear. Data were collected at a sampling rate of $1,000 \mathrm{~Hz}$ with the BrainAmp system (Brain Products $\mathrm{GmbH}$, Munich, Germany) and were analyzed offline. The obtained data were re-referenced to the connected mastoids (TP9-TP10). A $0.5 \mathrm{~Hz}$ low-cutoff as well as a $30 \mathrm{~Hz}$ high-cutoff filter was used. In addition to automatic artifact detection $(200 \mu \mathrm{V}$ 
amplitude criterion) all epochs were visually inspected, and those containing eye blinks or muscle movement artifacts were excluded. Baseline correction was performed on the basis of the $100 \mathrm{~ms}$ prestimulus interval. The amplitudes of $\mathrm{N} 1$ (referring to Fz) and N2-P2 (referring to TP9-TP10) components were measured.

Although we recorded data on 64 channels, we assessed LEPs according to the scalp distribution on the analyzed peaks. The N2 is a negative component (referring to TP9TP10) was peaking around 160-240 ms. The amplitude of the early $\mathrm{N} 1$ negative peak which came before $\mathrm{N} 2$ on $\mathrm{T} 7$ and T8 channels (referring to Fz) was analyzed. The P2 positive component after N2 was peaking around 300$360 \mathrm{~ms}$. The N2 component is largest over the lateral temporal and fronto-central areas on electrodes $\mathrm{Fz}, \mathrm{Cz}, \mathrm{CPz}$, F1-F4, FC1-FC6, C1-C6, T7, T8, and CP1-CP6. In contrast, the $\mathrm{P} 2$ peak has its maximum amplitude over the vertex on electrodes FCz, Cz, CPz, Pz, F1-F4, FC1-FC4, C1C4, CP1-CP4, and P1-P4. For the analysis of LEPs according to regional distribution, we defined three distinct areas with pooling the data: central (with all the midline electrodes such as $\mathrm{Fz}, \mathrm{FCz}, \mathrm{Cz}, \mathrm{CPz}$, and Pz), left (F3, FC3, C3, CP3, P3, FC5, C5, CP5, and T7) and right (electrodes according to the left side) instead of separate electrodes.

\section{Experimental design}

The subjects were sitting in a reclining chair. In case of all sessions first the EEG cap was placed on the head. After pain threshold determination the baseline LEP measurements were performed. Every run for the LEP recording consisted of 40 epochs of laser stimulation on each hand. The interstimulus interval of the stimulation ranged from 8 to $15 \mathrm{~s}$ (Raij et al. 2003). Thus the LEP recording lasted for 8-10 min for the first hand and also for 8-10 min for the second hand laser-stimulation. In all three TBS and sham TBS conditions, the right hand was stimulated first in half of the cases and the left hand was stimulated first in the other half. This order was kept for the subjects for all conditions. After baseline LEP recording the AMT was measured ( 15-20 min) and the TBS were applied for 40$190 \mathrm{~s}$ through the cap. After TBS the impedance of the EEG electrodes were retested and corrected below $5 \mathrm{k} \Omega$ if it was necessary. The TBS was followed by a post-stimulation LEP recording in $\sim 5 \mathrm{~min}$ after TBS. Thus, the interval between the two LEP recordings with regard to the same hand was about 30-35 min including all subjects and conditions.

The experimental sessions were separated from each other by at least 5 days. The subjects were blinded as to the type of magnetic stimulation. The order of the sessions was randomized across subjects.
Data analysis

The individually averaged VAS values and LEP amplitudes were entered into a repeated-measures-ANOVA for both hands and LEP components separately [four TBS CONDITION (cTBS, iTBS, imTBS, and sham) $\times 2$ TIME (before, after TBS)]. We considered a psychophysical or an electrophysiological change only if the CONDITION $\times$ TIME interaction was significant. In case of the LEP components we investigated if this effect was dependent on the defined areas by calculating the CONDITION $\times$ TIME $\times$ REGION interaction. Post hoc analysis was done using Student's $t$ tests (paired samples, two-tailed, level of significance $P<0.05)$.

\section{Results}

Psychophysics

The intensity of the laser stimulation $(1.4-1.6 \times$ of the pain threshold) was $19.88 \mathrm{~mJ} / \mathrm{mm}^{2}$ for cTBS, $20.53 \mathrm{~mJ} / \mathrm{mm}^{2}$ for iTBS, $20.52 \mathrm{~mJ} / \mathrm{mm}^{2}$ for imTBS, and $20.33 \mathrm{~mJ} / \mathrm{mm}^{2}$ for sham stimulation. None of the subjects reported any sideeffect after the stimulation.

In case of the contralateral hand (right) stimulation, repeated-measures-ANOVA revealed no main effect of CONDITION $[F(3,33)=0.828, P=0.488]$ but the TIME was significant $[F(1,11)=27.270, \quad P<0.001]$. The CONDITION $\times$ TIME interaction was also not significant $[F(3,33)=0.080, P=0.97]$. In case of the ipsilateral hand (left) stimulation, there was no significant main effect of CONDITION $[F(3,33)=1.329, P=0.282]$ but the TIME was significant $[F(1,11)=15.395, \quad P<0.005]$. The CONDITION $\times$ TIME interaction was also not significant $[F(3,33)=0.716, P=0.55]$ (Fig. 2).

Electrophysiology

The N1, N2, and P2 components could clearly be identified in all subjects. The LEPs are presented in Figs. 3 and 4.

\section{The N1 component}

We analyzed the amplitudes of the early N1 components on channels T7 and T8 (referring to Fz). There was no significant main effect of CONDITION [contralateral hand: $F(3,33)=2.216, \quad P=0.105 ;$ ipsilateral hand: $F(3,33)=$ $2.865, P=0.052]$. The TIME was significant if the contralateral hand was stimulated $[F(1,11)=6.186, P=0.030]$ but not for the ipsilateral hand $[F(1,11)=3.305$, $P=0.096]$. The interaction of the CONDITION $\times$ TIME resulted in no significant interaction neither after the 


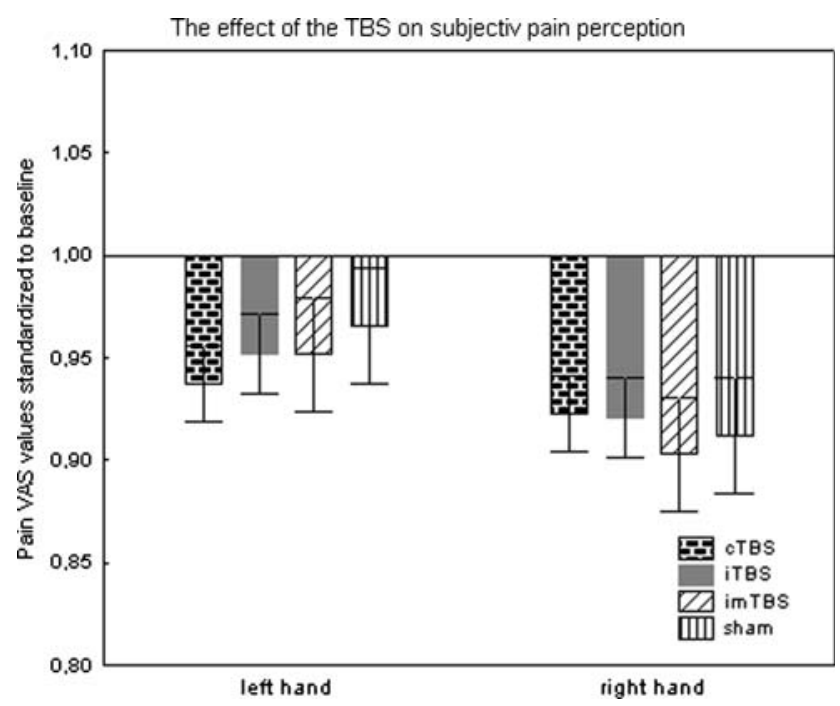

Fig. 2 The effects of the TBS on subjective pain perception. The VAS-values were standardized for each subject, for each condition by calculating the after/before ratio. The independent variables were the CONDITION and HAND in order to determine if there is any difference between stimulation of the two hands. There was no main effect of the stimulation CONDITION and the HAND (left or right). The interaction CONDITION $\times$ TIME was also not significant

contraleteral $[F(3,33)=0.727, P=0.543]$ or ipsilateral $[F(3,33)=1.694, P=0.187]$ hand stimulation (Fig. 3).

\section{The N2 component}

The repeated-measures-ANOVA (ipsilateral hand) showed no significant main effect of CONDITION $[F(3,33)=0.555, P=0.65]$, but the effect of TIME was significant $[F(1,11)=11.769, P=0.006]$. The CONDITION $\times$ TIME interaction resulted in no significance $[F(3,33)=$ $0.149, P=0.93$ ] (Fig. 5a). In case of the (contralateral hand) the main effect of CONDITION $[F(3,33)=0.250$, $P=0.86]$ was not significant, but the TIME was significant $[F(1,11)=32.034, P<0.001]$. The CONDITION $\times$ TIME interaction was also significant $[F(3,33)=4.058$, $P=0.015]$ (Fig. 5b). The interaction with electrode position was not significant $[F(6,66)=1.068, P=0.39]$. The post hoc $t$ test showed that all active TBS stimulation significantly decreased the amplitudes of the $\mathrm{N} 2$ component at all defined regions for the contralateral hand stimulation. Table 1 summarizes the results of $t$ tests.

\section{The P2 component}

The repeated-measures-ANOVA showed no significant main effect of CONDITION [contralateral: $F(3,33)=1.571, \quad P=0.22 ; \quad$ ipsilateral: $\quad F(3,33)=1.054$, $P=0.38$ ], but the TIME was significant [contralateral: $F(1,11)=17.038, P=0.002$; ipsilateral: $F(1,11)=15.362$, $P=0.002]$. The CONDITION $\times$ TIME interaction was also not significant [contralateral: $\quad F(3,33)=2.669$, $P=0.064$; ipsilateral: $F(3,33)=0.418, P=0.74]$.

The means of the different LEP components from all 12 subjects are presented in Table 2 .

\section{Discussion}

The main finding of our study is that all theta burst paradigms over the SI were able to diminish the amplitude of the N2 component of LEPs significantly when compared to sham stimulation. Surprisingly, the imTBS condition, that is suggested to be used as a placebo condition, when it applied over the M1 (Huang et al. 2005), also caused a strong amplitude decrease. The N1 and P2 components and the subjective pain rating scores were not significantly influenced by any type of TBS.

Recent studies using the theta burst paradigm have concentrated on the effects of continuous (cTBS) and intermittent theta burst stimulation (iTBS) (Franca et al. 2006; Andoh et al. 2007; Ishikawa et al. 2007; Koch et al. 2007; Mochizuki et al. 2007). None of them investigated the intermediate (imTBS) pattern since Huang and et al. published that it has no effect over the M1 as revealed by MEPs and could thus be used as a sham condition (Huang et al. 2005).

According to our knowledge, only five studies applied TBS over non-motor cortical areas. First Franca et al. (2006) used the theta burst pattern of rTMS over the visual cortex. They found that cTBS increased phosphene thresholds whilst iTBS was found to be ineffective. In another study, both cTBS and iTBS over the left dorsal premotor cortex decreased the transcallosal inhibition revealed by pairs of transcranial magnetic stimuli (Koch et al. 2007). In a recent study, Wernicke's area was stimulated with iTBS while the reaction time of auditory word detection was measured (Andoh et al. 2007). In this work, iTBS facilitated the detection of foreign words when compared with native words.

Concerning the SI, a shorter form of the iTBS (300 pulses) over the left SI resulted in a significant oxy-hemoglobin decrease at the contralateral SI and M1, detected by near infrared spectroscopy (Mochizuki et al. 2007). In another study cTBS of the SI resulted in a temporary decrease (13 min), whereas stimulation of the M1 caused a long-lasting increase (up to $53 \mathrm{~min}$ ) of the amplitudes of cortical components of the median nerve SEPs (Ishikawa et al. 2007). In summary, these results suggest that cTBS has an inhibitory effect on non-motor areas; whereas the effect of iTBS is more facilitatory, but clearer results have still to emerge. Our results are the first demonstrating that all three TBS paradigms, but not by sham stimulation over the SI resulted in similar after-effects regarding the ampli- 
Fig. 3 The effects of the TBS on the N1 component. The figure shows $\mathrm{N} 1$ amplitude changes during the experimental sessions on the grand averages of LEPs. The N1 was analyzed over the electrodes $\mathrm{T} 7$ and $\mathrm{T} 8$ referred to $\mathrm{Fz}$ (international 10-20 electrode system) in case of both hands. The solid line shows LEPs before and the intermittent line after TBS interventions. There was no significant difference between stimulation conditions

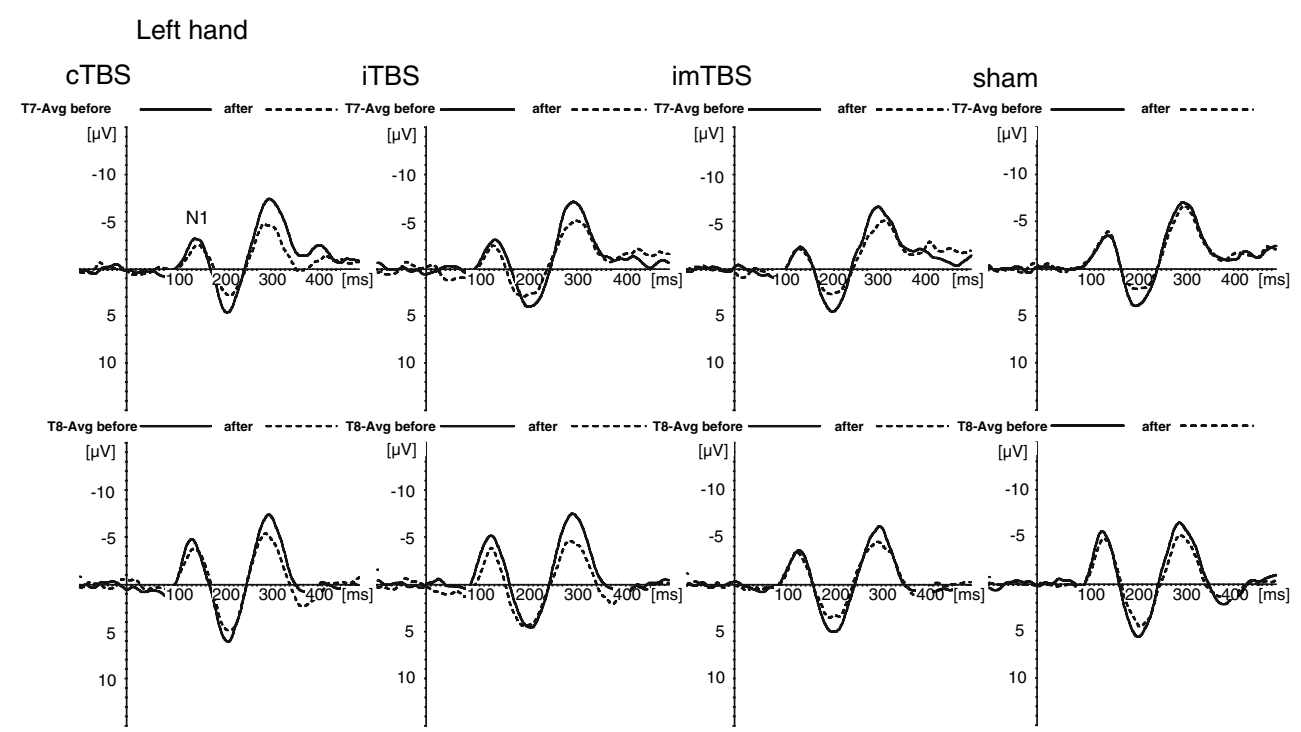

Right hand

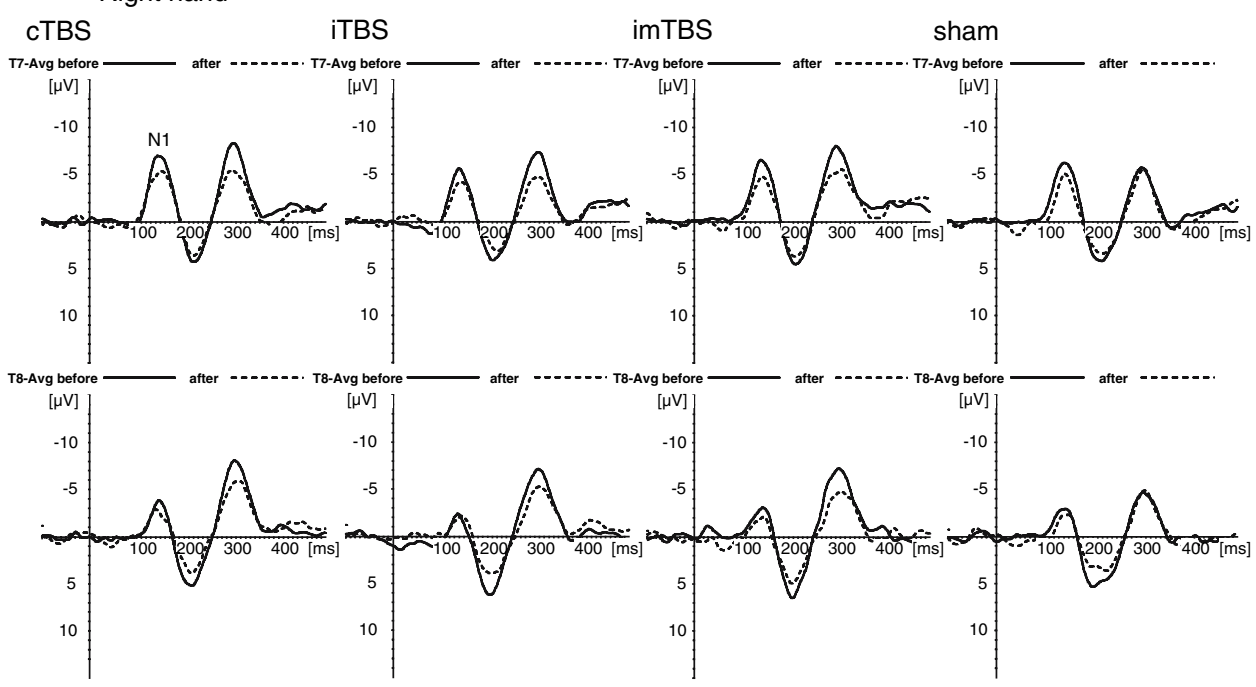

tude of the N2 component of LEPs evoked by the laser stimulation of the contralateral hand.

The N2 component (peaking around 160-220 ms), is generated bilaterally in the operculoinsular region and in the anterior cingulate cortex (ACC) (Garcia-Larrea et al. 2003) and reflects sensory, discriminatory processes (Garcia-Larrea et al. 1997; Iannetti et al. 2005); whilst the P2 component (peaking around 300-360 ms) arose mostly from the ACC and represents attentional, cognitive and affective factors of pain perception and processing (Treede 2003). However, other studies did not find significantly different brain sources for $\mathrm{N} 2$ and $\mathrm{P} 2$, revealing both parasylvian and ACC contributions for the N2-P2 components (Raij et al. 2003; Ohara et al. 2004). Thus, LEP changes in $\mathrm{N} 2$ or P2 component might result from changes in either sensory-discriminative or cognitive aspects of pain. Studies using subdural recordings (Kanda et al. 2000; Ohara et al. 2004) or MEG (Kanda et al. 2000) demonstrated that the
LEP components can be recorded over the SI and SII simultaneously, and the N2 peak may indicate the arrival of input originating from nociceptors.

With regard to the N1 component which is an early LEP potential (peaking around 140-170 ms) and reflecting the early sensory-discriminative processing of pain perception (Iannetti et al. 2005), we did not find any significant change in amplitude. According to scalp topography (maximum near T3 and T4), the N1 is generated near to the SII in the fronto-parietal operculum (Treede et al. 2003). The participation of operculoinsular cortex in coding the pain intensity was recently suggested by using LEP measurements (Ianetti et al. 2005). However, another study (Gracia-Larrea et al. 1997) did not find any significant correlation between the amplitude of the $\mathrm{N} 1$ component and subjective pain rating.

The fact that we found a decrement of both the N2 and P2 amplitudes in the sham condition as well, should be 


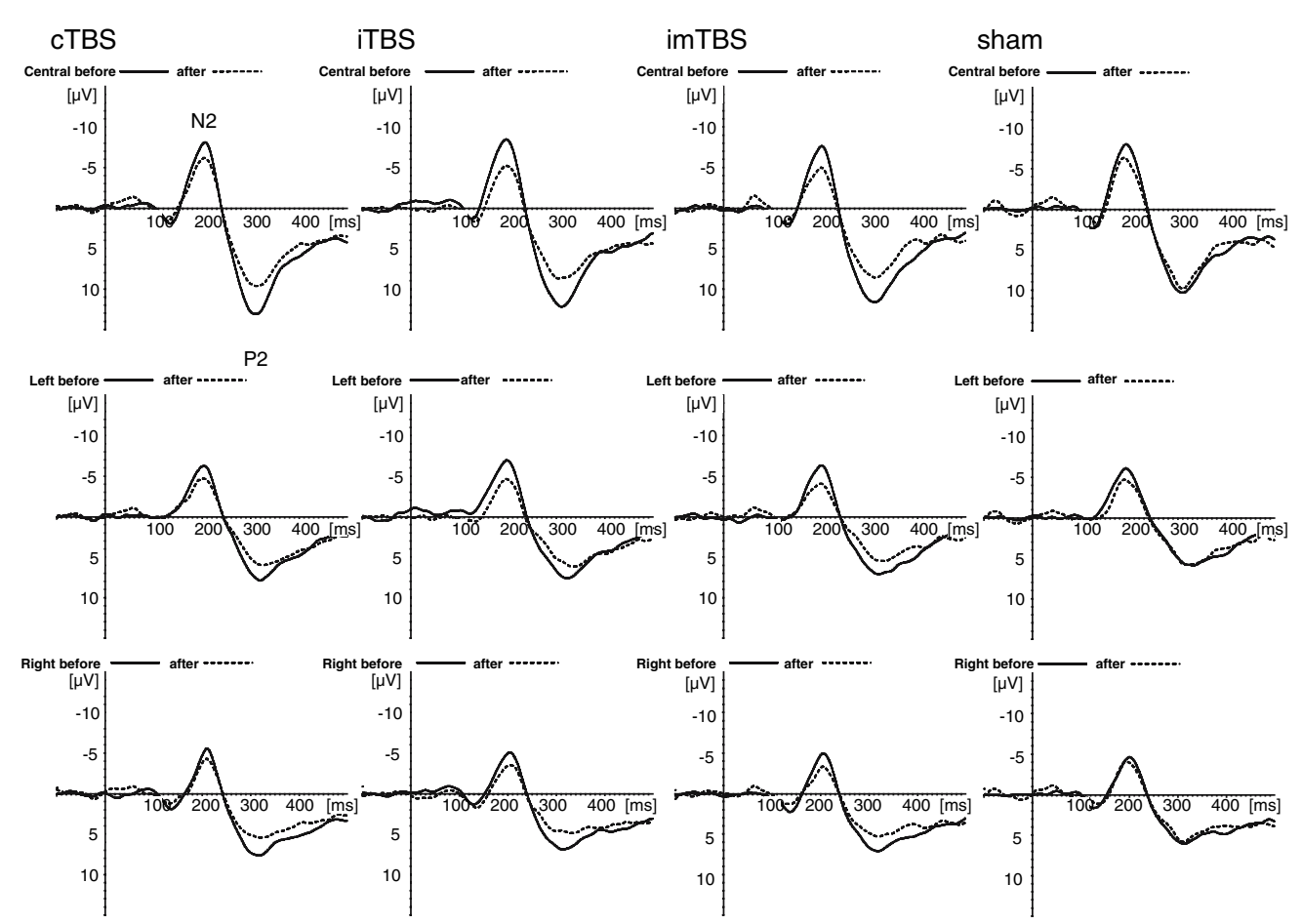

Fig. 4 Grand averages of LEPs obtained by contralateral (right) hand laser stimulation for three scalp regions (central, left and right) re-referenced to the connected mastoids (TP9-TP10), before and after the

three TBS conditions and sham stimulation. The solid line shows LEPs before and the intermittent line after TBS interventions

discussed. This phenomenon is known as habituation and has been described in previous LEP studies (Spiegel et al. 2000; Tamura et al. 2004a). Still, the effects of real TBS conditions on $\mathrm{N} 2$ amplitudes were greater than that of the sham condition and it was significant above the stimulated left, the neighboring central and the contralateral area as well when the right hand was laser stimulated. Attention can also directly influence the N2-P2 components of LEPs as well as subjective pain rating as it was suggested by previous experimental results (Gracia-Larrea et al. 1997; Ohara et al. 2006). In our experiment the subjects were asked to pay attention to each laser stimuli and since the TBS and sham condition were applied in a randomized order and the subjects were blinded as to the type of magnetic stimulation, the significant difference between sham and the other three TBS on the N2 amplitude is more than simply habituation or the effect of the different attentional states.

The possible origin of the $\mathrm{N} 2$ component is mainly the bilateral operculoinsular region and the ACC (GarciaLarrea et al. 2003). Therefore, when we inhibited the left SI, the activity of the pain related cortical network decreased due to the widespread cortical connections between SI and other cortical areas. However, the involvement of the contralateral SI and bilateral SII-parasylvian region in the N2 component generation was also reported (Kakigi et al. 2005; Kanda et al. 2000; Ohara et al. 2004). According to this, it is also possible that the inhibition of the SI itself may cause direct effects on LEP components. In this study we found no significant difference between the stimulation conditions with regard to subjective pain perception (Fig. 2), however, the subjective pain rating decreased during the experimental sessions after every type of TBS including the sham condition. It is important to mention that the placebo effect is high in almost every pain perception study, regardless of the paradigm used (Khedr et al. 2005; Lefaucheur et al. 2004). The explanation of this discrepancy between the electrophysiological and psychophysical changes is rather speculative, because the relationship between the anatomical origin of the N2-P2 components and their psychophysical correlates controversially discussed in the literature. In a PET study different cortical activations for pain threshold, intensity and unpleasantness have been found (Tölle et al. 1999). One possibility is that modulation the excitability of the left SI by TBS activated some elements of the pain related network that caused a decrease in the N2 amplitude. However, this stimulation intensity or duration was not strong or adequate enough to modify the subjective pain rating.

In our previous study (Antal et al. 2007), we found that cathodal tDCS over the SI, similarly to the present findings, significantly decreased the N2 component of LEPs. In contrast to the present findings the subjective pain perception in healthy subjects was also diminished. TDCS is a method 

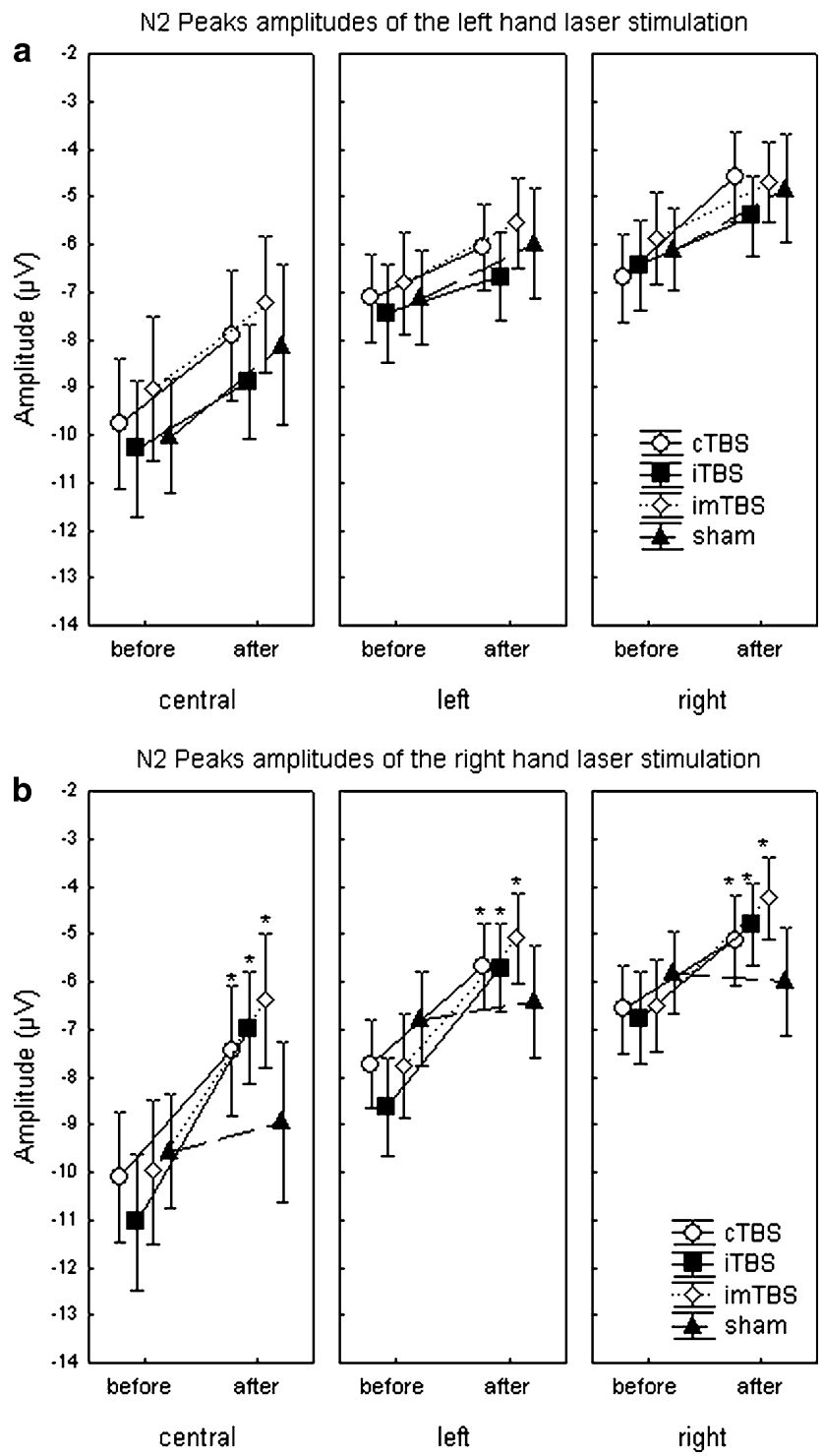

Fig. 5 The mean N2 amplitude changes during the four TBS conditions at the ipsilateral (left) (Fig. 5a) and contralateral (right) (Fig. 5b) hand laser-stimulation for the three calculated regions (central, left and right). The stars mark significant differences between before-after TBS conditions (post hoc $t$-tests, paired samples, two-tailed, $P<0.05$ ) that modifies the resting membrane potentials of cortical neurons intracortically (Nitsche and Paulus 2000, 2001). Cathodal stimulation decreases, whilst anodal stimulation increases cortical excitability (Nitsche and Paulus 2000, 2001). However, in that study we have used a large electrode size $(5 \times 7 \mathrm{~cm})$ in order to optimize stimulation's parameters (Nitsche and Paulus 2000) and therefore we might have covered a large part of the SI. It is possible that we have stimulated one part of the somatosensory association cortex (BA 5/7) that is posterior to the SI. Activation of human BA5/7 has also been linked to pain perception (Apkarian et al. 1999; Forss et al. 2005). BA 5/7 is anatomically connected to other nociceptive brain areas such as the ACC, insula, thalamus and primary motor cortices (Friedman et al. 1986). In order to increase the focality of the transcranial stimulation in the present study we used a neuronavigation system to determine the hand area over the SI.

Many of the previous pain-related studies stimulated the M1. The effects of low and high frequency rTMS of M1 on experimentally induced acute pain perception seem to depend on the type of noxious stimulation. C-fiber mediated acute pain as induced by intradermal capsaicin administration could be attenuated by $1 \mathrm{~Hz}$ rTMS over the M1 (Tamura et al. 2004b), whereas it increased $\mathrm{A} \delta$-fiber mediated laser-induced pain in another study (Tamura et al. 2004a). Similarly, controversial effects were observed after $20 \mathrm{~Hz}$ rTMS (Johnson et al. 2006; Summers et al. 2004). In contrast $10 \mathrm{~Hz}$ rTMS over M1 increased electrically induced $\mathrm{A} \delta$-fiber mediated pain threshold (Yoo et al. 2006), but others found that $10 \mathrm{~Hz}$ rTMS has only an effect on the unpleasantness of the pain without any effect on pain threshold (Mylius et al. 2007). In case of chronic pain the high frequency rTMS seems to more effective than the application of low frequencies (Leo and Latif 2007).

The neuronal mechanism of the theta burst paradigm is highly speculative. The results of the experiments with single trains of TBS suggest that in the human M1 TBS produces a mixture of facilitatory and inhibitory effects on synaptic transmission (Huang and Rothwell 2004). Huang
Table 1 Post hoc analysis of the N2 component

Shows the results of the Student's $t$-tests (paired samples, two-tailed) in case of the right hand stimulation. The level of the significance was $P<0.05$

\begin{tabular}{lllllc}
\hline & cTBS & iTBS & imTBS & sham \\
& Before versus & $\begin{array}{l}\text { Before versus } \\
\text { after }\end{array}$ & $\begin{array}{l}\text { Before versus } \\
\text { after }\end{array}$ & $\begin{array}{l}\text { Before versus } \\
\text { after }\end{array}$ \\
\hline \multirow{2}{*}{ Central } & $P$-levels & 0.0033 & 0.0002 & 0.0021 & 0.3942 \\
& $t$-values & -3.730 & -5.499 & -4.005 & -0.887 \\
\multirow{2}{*}{ Reft } & $P$-levels & 0.0054 & 0.0004 & 0.0031 & 0.5921 \\
& $t$-values & -3.451 & -5.041 & -3.775 & -0.552 \\
& $P$-levels & 0.0394 & 0.0085 & 0.0039 & 0.8118 \\
& $t$-values & -2.337 & -3.196 & -3.645 & 0.244 \\
\hline
\end{tabular}


Table 2 The mean amplitudes of the LEP components

\begin{tabular}{|c|c|c|c|c|c|c|c|c|c|}
\hline \multirow[t]{2}{*}{ Peak } & & \multicolumn{2}{|l|}{ cTBS } & \multicolumn{2}{|l|}{ iTBS } & \multicolumn{2}{|l|}{ imTBS } & \multicolumn{2}{|l|}{ sham } \\
\hline & & Before & After & Before & After & Before & After & Before & After \\
\hline \multicolumn{10}{|c|}{ Left hand } \\
\hline \multirow[t]{2}{*}{ N1 } & $\mathrm{T} 7$ & $-4.42 \pm 2.75$ & $-4.60 \pm 3.13$ & $-4.68 \pm 2.52$ & $-3.71 \pm 2.65$ & $-3.33 \pm 3.22$ & $-3.50 \pm 3.15$ & $-4.98 \pm 2.70$ & $-5.20 \pm 3.08$ \\
\hline & $\mathrm{T} 8$ & $-6.13 \pm 2.17$ & $-4.78 \pm 3.60$ & $-6.73 \pm 3.44$ & $-5.13 \pm 2.89$ & $-4.99 \pm 4.27$ & $-4.64 \pm 3.62$ & $-6.56 \pm 3.47$ & $-6.33 \pm 3.11$ \\
\hline \multirow[t]{3}{*}{$\mathrm{N} 2$} & Central & $-9.77 \pm 5.48$ & $-7.92 \pm 4.91$ & $-10.29 \pm 5.01$ & $-8.88 \pm 3.98$ & $-9.04 \pm 5.43$ & $-7.25 \pm 5.52$ & $-10.02 \pm 4.43$ & $-8.11 \pm 6.16$ \\
\hline & Left & $-7.13 \pm 3.60$ & $-6.05 \pm 3.44$ & $-7.44 \pm 3.49$ & $-6.69 \pm 2.91$ & $-6.81 \pm 3.69$ & $-5.55 \pm 3.88$ & $-7.13 \pm 3.55$ & $-5.98 \pm 4.28$ \\
\hline & Right & $-6.70 \pm 3.05$ & $-4.59 \pm 3.25$ & $-6.42 \pm 3.54$ & $-5.41 \pm 2.56$ & $-5.88 \pm 3.05$ & $-4.70 \pm 3.19$ & $-6.11 \pm 3.24$ & $-4.82 \pm 3.85$ \\
\hline \multirow[t]{3}{*}{$\mathrm{P} 2$} & Central & $14.79 \pm 8.02$ & $11.74 \pm 7.03$ & $14.86 \pm 7.69$ & $12.43 \pm 7.00$ & $13.19 \pm 7.74$ & $11.26 \pm 8.23$ & $13.83 \pm 6.75$ & $12.03 \pm 6.31$ \\
\hline & Left & $8.40 \pm 3.89$ & $7.18 \pm 3.66$ & $9.19 \pm 4.74$ & $7.74 \pm 3.59$ & $7.36 \pm 4.80$ & $6.63 \pm 5.16$ & $7.90 \pm 3.64$ & $6.84 \pm 3.67$ \\
\hline & Right & $8.80 \pm 3.80$ & $6.78 \pm 3.03$ & $8.68 \pm 4.65$ & $7.59 \pm 3.68$ & $7.91 \pm 3.70$ & $6.90 \pm 3.98$ & $8.28 \pm 4.27$ & $7.76 \pm 4.17$ \\
\hline \multicolumn{10}{|c|}{ Right hand } \\
\hline \multirow[t]{2}{*}{$\mathrm{N} 1$} & $\mathrm{~T} 7$ & $-8.18 \pm 4.79$ & $-5.98 \pm 3.39$ & $-6.38 \pm 4.67$ & $-5.12 \pm 3.62$ & $-7.34 \pm 4.70$ & $-5.88 \pm 3.73$ & $-7.43 \pm 3.30$ & $-6.38 \pm 3.21$ \\
\hline & $\mathrm{T} 8$ & $-4.92 \pm 4.43$ & $-3.96 \pm 3.24$ & $-3.25 \pm 3.29$ & $-2.98 \pm 2.35$ & $-4.56 \pm 4.61$ & $-3.03 \pm 2.39$ & $-4.37 \pm 3.53$ & $-4.15 \pm 2.62$ \\
\hline \multirow[t]{3}{*}{ N2 } & Central & $-10.11 \pm 3.83$ & $-7.44 \pm 4.52$ & $-11.05 \pm 4.87$ & $-6.69 \pm 4.23$ & $-9.99 \pm 5.06$ & $-6.40 \pm 4.27$ & -9.573 .97 & $-8.94 \pm 5.40$ \\
\hline & Left & $-7.74 \pm 2.70$ & $-5.67 \pm 2.77$ & $-8.62 \pm 3.65$ & $-5.71 \pm 3.43$ & $-7.77 \pm 3.80$ & $-5.09 \pm 2.64$ & $-6.79 \pm 3.29$ & $-6.42 \pm 3.78$ \\
\hline & Right & $-6.58 \pm 3.37$ & $-5.12 \pm 3.34$ & $-6.76 \pm 3.00$ & $-4.80 \pm 3.26$ & $-6.52 \pm 3.59$ & $-4.24 \pm 2.72$ & $-5.82 \pm 2.74$ & $-6.00 \pm 3.99$ \\
\hline \multirow[t]{3}{*}{$\mathrm{P} 2$} & Central & $14.30 \pm 6.56$ & $11.62 \pm 5.93$ & $14.52 \pm 7.14$ & $10.90 \pm 5.49$ & $14.04 \pm 7.79$ & $10.74 \pm 7.13$ & $11.37 \pm 6.38$ & $10.75 \pm 5.73$ \\
\hline & Left & $8.47 \pm 3.87$ & $6.95 \pm 3.03$ & $8.90 \pm 4.14$ & $7.00 \pm 2.91$ & $8.65 \pm 4.79$ & $6.64 \pm 3.37$ & $6.21 \pm 3.45$ & $6.62 \pm 3.22$ \\
\hline & Right & $8.52 \pm 3.83$ & $6.73 \pm 2.75$ & $8.29 \pm 4.17$ & $6.41 \pm 3.32$ & $8.75 \pm 4.29$ & $6.90 \pm 3.76$ & $6.84 \pm 4.44$ & $6.67 \pm 4.04$ \\
\hline
\end{tabular}

The mean amplitudes of the LEP components before and after stimulation in all four conditions. (mean \pm standard deviation)

and Rothwell proposed, that facilitation develops faster than inhibition, thus in case of the inhibitory cTBS, several seconds after an initial facilitation the inhibition overrides this effect. ITBS uses only the early excitatory effect in the initial $2 \mathrm{~s}$ and after this the stimulation is interrupted for $8 \mathrm{~s}$. Most likely, the underlying mechanisms will involve many of the basic elementary mechanisms described previously in the LTP/LTD literature (Paulus 2005). Recently Huang et al. (2007) have demonstrated that the after-effects produced by both iTBS or cTBS are NMDA-receptor dependent and hence they are more likely to involve plasticity-like changes at the synapse in the M1. More recently, it was found that the excitatory effects of iTBS were reversed after NMDA receptor activation by D-cycloserin (Teo et al. 2007). This is in contrast with the findings of tDCS induced LTP where the excitatory effects are prolonged by D-cycloserin (Nitsche et al. 2004). However, it is important to note that these observations were done on the M1. It is possible that the different effectiveness observed between TBS protocols on motor and sensory cortices could be due to differences in the physiological and functional states of the stimulated cortex.

In summary, in our study we found a significant decrease of the N2 component of the LEPs after cTBS, iTBS, and imTBS when compared to sham stimulation over the SI. In addition we found, that the subjective pain perception did not show significant differences among the stimulation conditions suggesting that this method is probably not the most effective in decreasing subjective pain perception. Further findings show that imTBS resulted in more impressive modification of the LEPs, than were found in previous TMS-studies over the M1 using MEP measures (Huang et al. 2005), implying that it should not be used in further studies as 'sham' condition, at least with regard to LEPs and when it is applied over the SI. However these results are not directly comparable with the effects of TBS on M1, thus further studies are needed to clarify the effects of imTBS on different cortical areas. Future studies should also clarify the effectiveness of the different TBS paradigms applied over the M1 and non-motor cortical areas, such as the SII on acute pain perception and in chronic pain.

Acknowledgments We would like to thank Leila Chaieb for the English corrections and Prof. RD. Treede for his useful suggestions with regard to the technical details. This study was supported by the German Ministry of Research and Education within the "Kompetenznetz Schmerz" (FKZ: 01EM0117) and by Waler und Ilse Rose-Stiftung.

Open Access This article is distributed under the terms of the Creative Commons Attribution Noncommercial License which permits any noncommercial use, distribution, and reproduction in any medium, provided the original author(s) and source are credited.

\section{References}

Andoh J, Artiges E, Pallier C, Riviere D, Mangin JF, Paillere-Martinot ML, Martinot JL (2007) Priming frequencies of transcranial 
magnetic stimulation over Wernicke's area modulate word detection. Cereb Cortex (in press). doi:10.1093/cercor/bhm047

Antal A, Brepohl N, Poreisz Cs, Boros K, Csifcsák G, Paulus W (2007) Transcranial direct current stimulation over somatosensory cortex decreases experimentally induced acute pain perception. Clin J Pain (in press)

Apkarian AV, Darbar A, Krauss BR, Gelnar PA, Szeverenyi NM (1999) Differentiating cortical areas related to pain perception from stimulus identification: temporal analysis of fMRI activity. J Neurophysiol 81:2956-2963

Apkarian AV, Bushnell MC, Treede RD, Zubieta JK (2005) Human brain mechanisms of pain perception and regulation in health and disease. Eur J Pain 9:463-484

Blankenburg F, Ruben J, Meyer R, Schwiemann J, Villringer A (2003) Evidence for a rostral-to-caudal somatotopic organization in human primary somatosensory cortex with mirror-reversal in areas $3 \mathrm{~b}$ and 1. Cereb Cortex 13:987-993

Bushnell MC, Duncan GH, Hofbauer RK, Ha B, Chen JI, Carrier B (1999) Pain perception: is there a role for primary somatosensory cortex? Proc Natl Acad Sci USA 96:7705-7709

Casey KL, Minoshima S, Berger KL, Koeppe RA, Morrow TJ, Frey KA (1994) Positron emission tomographic analysis of cerebral structures activated specifically by repetitive noxious heat stimuli. J Neurophysiol 71:802-807

Chen JI, Ha B, Bushnell MC, Pike B, Duncan GH (2002) Differentiating noxious- and innocuous-related activation of human somatosensory cortices using temporal analysis of fMRI. J Neurophysiol $88: 464-474$

Coghill RC, Sang CN, Maisog JM, Iadarola MJ (1999) Pain intensity processing within the human brain: a bilateral, distributed mechanism. J Neurophysiol 82:1934-1943

Di Lazzaro V, Pilato F, Saturno E, Oliviero A, Dileone M, Mazzone P, Insola A, Tonali PA, Ranieri F, Huang YZ, Rothwell JC (2005) Theta-burst repetitive transcranial magnetic stimulation suppresses specific excitatory circuits in the human motor cortex. J Physiol 565:945-950

Dieckhofer A, Waberski TD, Nitsche M, Paulus W, Buchner H, Gobbele R (2006) Transcranial direct current stimulation applied over the somatosensory cortex - differential effect on low and high frequency SEPs. Clin Neurophysiol 117:2221-2227

Enomoto H, Ugawa Y, Hanajima R, Yuasa K, Mochizuki H, Terao Y, Shiio Y, Furubayashi T, Iwata NK, Kanazawa I (2001) Decreased sensory cortical excitability after $1 \mathrm{~Hz}$ rTMS over the ipsilateral primary motor cortex. Clin Neuropysiol 112:2154-2158

Forss N, Raij TT, Seppa M, Hari R (2005) Common cortical network for first and second pain. Neuroimage 24:132-142

Franca M, Koch G, Mochizuki H, Huang YZ, Rothwell JC (2006) Effects of theta burst stimulation protocols on phosphene threshold. Clin Neurophysiol 117:1808-1813

Fregni F, Freedman S, Pascual-Leone A (2007) Recent advances in the treatment of chronic pain with non-invasive brain stimulation techniques. Lancet Neurol 6:188-191

Friedman DP, Murray EA, O’Neill JB, Mishkin M (1986) Cortical connections of the somatosensory fields of the lateral sulcus of macaques: evidence for a corticolimbic pathway for touch. J Comp Neurol 252:323-347

Garcia-Larrea L, Peyron R, Laurent B, Mauguiere F (1997) Association and dissociation between laser-evoked potentials and pain perception. Neuroreport 8:3785-3789

Garcia-Larrea L, Frot M, Valeriani M (2003) Brain generators of laserevoked potentials: from dipoles to functional significance. Neurophysiol Clin 33:279-292

Gelnar PA, Krauss BR, Sheehe PR, Szeverenyi NM, Apkarian AV (1999) A comparative fMRI study of cortical representations for thermal painful, vibrotactile, and motor performance tasks. Neuroimage 10:460-482
Huang YZ, Rothwell JC (2004) The effect of short-duration bursts of high-frequency, low-intensity transcranial magnetic stimulation on the human motor cortex. Clin Neurophysiol 115:1069-1075

Huang YZ, Edwards MJ, Rounis E, Bhatia KP, Rothwell JC (2005) Theta burst stimulation of the human motor cortex. Neuron 45:201-206

Huang YZ, Chen RS, Rothwell JC, Wen HY (2007) The after-effect of human theta burst stimulation is NMDA receptor dependent. Clin Neurophysiol 118:1028-1032

Inui K, Wang X, Qiu Y, Nguyen BT, Ojima S, Tamura Y, Nakata H, Wasaka T, Tran TD, Kakigi R (2003) Pain processing within the primary somatosensory cortex in humans. Eur $\mathrm{J}$ Neurosci 18:2859-2866

Ishikawa S, Matsunaga K, Nakanishi R, Kawahira K, Murayama N, Tsuji S, Huang YZ, Rothwell JC (2007) Effect of theta burst stimulation over the human sensorimotor cortex on motor and somatosensory evoked potentials. Clin Neurophysiol 118:1033-1043

Johnson S, Summers J, Pridmore S (2006) Changes to somatosensory detection and pain thresholds following high frequency repetitive TMS of the motor cortex in individuals suffering from chronic pain. Pain 123:187-192

Kakigi R, Inui K, Tamura Y (2005) Electrophysiological studies on human pain perception. Clin Neurophysiol 116:743-763

Kanda M, Nagamine T, Ikeda A, Ohara S, Kunieda T, Fujiwara N, Yazawa S, Sawamoto N, Matsumoto R, Taki W, Shibasaki H (2000) Primary somatosensory cortex is actively involved in pain processing in human. Brain Res 853:282-289

Kanda M, Mima T, Oga T, Matsuhashi M, Toma K, Hara H, Satow T, Nagamine T, Rothwell JC, Shibasaki H (2003) Transcranial magnetic stimulation (TMS) of the sensorimotor cortex and medial frontal cortex modifies human pain perception. Clin Neurophysiol 114:860-866

Khedr EM, Kotb H, Kamel NF, Ahmed MA, Sadek R, Rothwell JC (2005) Longlasting antalgic effects of daily sessions of repetitive transcranial magnetic stimulation in central and peripheral neuropathic pain. J Neurol Neurosurg Psychiatry 76:833-838

Koch G, Franca M, Mochizuki H, Marconi B, Caltagirone C, Rothwell JC (2007) Interactions between pairs of transcranial magnetic stimuli over the human left dorsal premotor cortex differ from those seen in primary motor cortex. J Physiol 15:551-562

Kujirai T, Sato M, Rothwell JC, Cohen LG (1993) The effect of transcranial magnetic stimulation on median nerve somatosensory evoked potentials. Electroencephalogr Clin Neurophysiol 89:227-234

Lefaucheur JP, Drouot X, Menard-Lefaucheur I, Zerah F, Bendib B, Cesaro P, Keravel Y, Nguyen JP (2004) Neurogenic pain relief by repetitive transcranial magnetic cortical stimulation depends on the origin and the site of pain. J Neurol Neurosurg Psychiatry 75:612-616

Leo RJ, Latif T (2007) Repetitive transcranial magnetic stimulation (rTMS) in experimentally induced and chronic neuropathic pain: a review. J Pain 8:453-459

Lisanby SH, Gutman D, Luber B, Schroeder C, Sackeim HA (2001) Sham TMS: intracerebral measurement of the induced electrical field and the induction of motor-evoked potentials. Biol Psychiatry 49:460-463

Matsunaga K, Nitsche MA, Tsuji S, Rothwell JC (2004) Effect of transcranial DC sensorimotor cortex stimulation on somatosensory evoked potentials in humans. Clin Neurophysiol 115:456460

Mochizuki H, Franca M, Huang YZ, Rothwell JC (2005) The role of dorsal premotor area in reaction task: comparing the "virtual lesion" effect of paired pulse or theta burst transcranial magnetic stimulation. Exp Brain Res 167:414-421

Mochizuki H, Furubayashi T, Hanajima R, Terao Y, Mizuno Y, Okabe S, Ugawa Y (2007) Hemoglobin concentration changes in the 
contralateral hemisphere during and after theta burst stimulation of the human sensorimotor cortices. Exp Brain Res 180:667-675

Mylius V, Reis J, Knaack A, Haag A, Oertel WH, Rosenow F, Schepelmann K (2007) High-frequency rTMS of the motor cortex does not influence the nociceptive flexion reflex but increases the unpleasantness of electrically induced pain. Neurosci Lett 415:49-54

Nakata H, Inui K, Wasaka T, Tamura Y, Tran TD, Qiu Y, Wang X, Nguyen BT, Kakigi R (2004) Movements modulate cortical activities evoked by noxious stimulation. Pain 107:91-98

Nitsche MA, Jaussi W, Liebetanz D, Lang N, Tergau F, Paulus W (2004) Consolidation of human motor cortical neuroplasticity by D-cycloserine. Neuropsychopharmacology 29:1573-1578

Nitsche MA, Paulus W (2000) Excitability changes induced in the human motor cortex by weak transcranial direct current stimulation. J Physiol 527:633-639

Nitsche MA, Paulus W (2001) Sustained excitability elevations induced by transcranial DC motor cortex stimulation in humans. Neurology 57:1899-1901

Ohara S, Crone NE, Weiss N, Treede RD, Lenz FA (2004) Cutaneous painful laser stimuli evoke responses recorded directly from primary somatosensory cortex in awake humans. J Neurophysiol 91:2734-2746

Ohara S, Anderson WS, Lawson HC, Lee HT, Lenz FA (2006) Endogenous and exogenous modulators of potentials evoked by a painful cutaneous laser (LEPs). Acta Neurochir Suppl 99:77-79

Paulus W (2005) Toward establishing a therapeutic window for rTMS by theta burst stimulation. Neuron 45:181-183

Peyron R, García-Larrea L, Grégoire MC, Costes N, Convers P, Lavenne F, Mauguière F, Michel D, Laurent B (1999) Haemodynamic brain responses to acute pain in humans: sensory and attentional networks. Brain 122:1765-1779

Peyron R, Laurent B, Garcia-Larrea L (2000) Functional imaging of brain responses to pain. A review and meta-analysis. Neurophysiol Clin 30:263-288

Ploner M, Schmitz F, Freund HJ, Schnitzler A (1999) Parallel activation of primary and secondary somatosensory cortices in human pain processing. J Neurophysiol 81:3100-3104

Ploner M, Schmitz F, Freund HJ, Schnitzler A (2000) Differential organization of touch and pain in human primary somatosensory cortex. J Neurophysiol 83:1770-1776

Ploner M, Gross J, Timmermann L, Schnitzler A (2002) Cortical representation of first and second pain sensation in humans. Proc Natl Acad Sci USA 99:12444-12448

Raij TT, Vartiainen NV, Jousmaki V, Hari R (2003) Effects of interstimulus interval on cortical responses to painful laser stimulation. J Clin Neurophysiol 20:73-79

Rogalewski A, Breitenstein C, Nitsche MA, Paulus W, Knecht S (2004) Transcranial direct current stimulation disrupts tactile perception. Eur J Neurosci 20:313-316

Spiegel J, Hansen C, Treede RD (2000) Clinical evaluation criteria for the assessment of impaired pain sensitivity by thulium-laser evoked potentials. Clin Neurophysiol 111:725-735
Summers J, Johnson S, Pridmore S, Oberoi G (2004) Changes to cold detection and pain thresholds following low and high frequency transcranial magnetic stimulation of the motor cortex. Neurosci Lett 368:197-200

Talbot JD, Marrett S, Evans AC, Meyer E, Bushnell MC, Duncan GH (1991) Multiple representations of pain in human cerebral cortex. Science 251:1355-1358

Tamura Y, Hoshiyama M, Inui K, Nakata H, Qiu Y, Ugawa Y, Inoue K, Kakigi R (2004a) Facilitation of A[delta]-fiber-mediated acute pain by repetitive transcranial magnetic stimulation. Neurology 62:2176-2181

Tamura Y, Okabe S, Ohnishi T, N Saito D, Arai N, Mochio S, Inoue K, Ugawa Y (2004b) Effects of 1-Hz repetitive transcranial magnetic stimulation on acute pain induced by capsaicin. Pain 107:107-115

Tarkka IM, Treede RD (1993) Equivalent electrical source analysis of pain-related somatosensory evoked potentials elicited by a $\mathrm{CO} 2$ laser. J Clin Neurophysiol 10:513-519

Teo JT, Swayne OB, Rothwell JC (2007) Further evidence for NMDAdependence of the after-effects of human theta burst stimulation. Clin Neurophysiol 118:1649-1651

Timmermann L, Ploner M, Haucke K, Schmitz F, Baltissen R, Schnitzler A (2001) Differential coding of pain intensity in the human primary and secondary somatosensory cortex. J Neurophysiol $86: 1499-1503$

Tölle TR, Kaufmann T, Siessmeier T, Lautenbacher S, Berthele A, Munz F, Zieglgansberger W, Willoch F, Schwaiger M, Conrad B, Bartenstein P (1999) Region-specific encoding of sensory and affective components of pain in the human brain: a positron emission tomography correlation analysis. Ann Neurol 45:40-47

Treede RD, Lorenz J, Baumgartner U (2003) Clinical usefulness of laser-evoked potentials. Neurophysiol Clin 33:303-331

Treede RD (2003) Neurophysiological studies of pain pathways in peripheral and central nervous system disorders. J Neurol 250:1152-1161

Tsuji T, Rothwell JC (2002) Long lasting effects of rTMS and associated peripheral sensory input on MEPs, SEPs and transcortical reflex excitability in humans. J Physiol 540:367-376

Valeriani M, Barba C, le Pera D, Restuccia D, Colicchio G, Tonali P, Gagliardo O, Treede RD (2004) Different neuronal contribution to N20 somatosensory evoked potential and to $\mathrm{CO} 2$ laser evoked potentials: an intracerebral recording study. Clin Neurophysiol 115:211-216

Wolters A, Schmidt A, Schramm A, Zeller D, Naumann M, Kunesch E, Benecke R, Reiners K, Classen J (2005) Timing-dependent plasticity in human primary somatosensory cortex. J Physiol 565:1039-1052

Yoo WK, Kim YH, Doh WS, Lee JH, Jung KI, Park DS, Park ES (2006) Dissociable modulating effect of repetitive transcranial magnetic stimulation on sensory and pain perception. Neuroreport $17: 141-144$ 